\title{
Estimation of the Kullback-Leibler Divergence for Canny Edge Detector of Optoelectronic Images Segmentation
}

\author{
Hennadii Khudov ${ }^{1}$, Rostyslav Khudov ${ }^{2}$, Irina Khizhnyak ${ }^{3}$, \\ Volodymyr Loza ${ }^{4}$, Taras Kravets ${ }^{5}$, Sergii Kibitkin ${ }^{6}$ \\ ${ }^{, 1}$ Department of Radar Troops Tactic, Ivan Kozhedub Kharkiv National Air Force University, Kharkiv, Ukraine, \\ 2345kh_hg@ukr.net \\ ${ }^{2}$ Department of Theoretical and Applied Informatics, Kharkiv National University named after V. N. Karazin, \\ Kharkiv, Ukraine, rhudov@gmail.com \\ ${ }^{3}$ Department of Mathematical and Software Automated Control Systems, Ivan Kozhedub Kharkiv National Air \\ Force University, Kharkiv, Ukraine, khizh_ia@ukr.net \\ ${ }^{4}$ Department of Communications and Automated Control Systems, Ivan Cherniakhovskyi National Defence \\ University, Kyiv, Ukraine, vladymyrloza@gmail.com \\ ${ }^{5}$ Department of Artillery Facility of Rocket Forces and Artillery, Hetman Petro Sahaidachnyi National Army \\ Academy, Lviv, Ukraine, 2345kh_hg@ukr.net \\ ${ }^{6}$ Department of Aviation equipment and aerial reconnaissance complexes, Ivan Kozhedub Kharkiv National Air \\ Force University, Kharkiv, Ukraine, sergejkibitkin@ukr.net
}

\begin{abstract}
The result of decoding the images obtained from on-board optical-electronic surveillance systems depends on the quality of image segmentation, especially taking into account the peculiarities of their production (heterogeneous background, variability of different parts of the image, the presence of noise). The main techniques, criteria and indicators of image segmentation, their advantages and disadvantages are analyzed. It is proposed to evaluate the information indicator (Kulbak-Leibner distance) of thematic segmentation of the optoelectronic image by the Canney method. The analysis of the main stages of the Cannes method is carried out: smoothing, gradient search, suppression of false maxima, double threshold filtering, tracing of the uncertainty region. The result of segmentation of the optoelectronic image by the Canney method is given, the calculation of the Kulbak-Leibner distance on its dependence on the scale factor of the original image is carried out.
\end{abstract}

Key words : the optoelectronic image, on-board surveillance system, segmentation, the Canny edge detection algorithm, the Kullback-Leibler divergence.

\section{INTRODUCTION}

In modern conditions of network-centric and hybrid wars and anti-terrorist operations, about $80 \%$ of reconnaissance missions, $60 \%$ of security tasks and $50 \%$ of fire assault tasks are solved using information obtained from on-board surveillance systems (unmanned aerial vehicles and space surveillance systems) [1]-[6].
The efficiency of image interpretation obtained from on-board surveillance systems can be represented by four categories [7]: "A" - confident interpretation without the use of additional materials; "B" - interpretation is possible in-house using additional materials; " $\mathrm{C}$ " - interpretation is possible only using field research; "D" - interpretation is not possible.

Table 1 shows the summary results of the possibility of interpretation of objects using optoelectronic images obtained from Pleiades, WorldView spacecraft (SC) and when performing aerial photography using the VisionMap A3 camera [8]. 236 objects of the following types were analyzed: land relief; hydrography; localities; socio-economic objects; road networks and road constructions; vegetation and soil.

Table 1: The summary results of the possibility of interpretation of objects

\begin{tabular}{|c|c|c|c|c|}
\hline \multirow{2}{*}{$\begin{array}{c}\text { Output data } \\
\text { type }\end{array}$} & \multicolumn{4}{|c|}{ Number of objects } \\
\cline { 2 - 5 } & A & B & C & D \\
\hline $\begin{array}{c}\text { SC } \\
\text { Pleiades }\end{array}$ & 103 & 92 & 21 & 20 \\
\hline $\begin{array}{c}\text { SC } \\
\text { WorldView }\end{array}$ & 106 & 90 & 19 & 21 \\
\hline $\begin{array}{c}\text { Camera } \\
\text { Vision A3 }\end{array}$ & 126 & 78 & 16 & 16 \\
\hline
\end{tabular}

From the analysis of the results shown in table 1, it can be seen that for slightly less than half of the objects they are interpreted with confidence (category "A"). Approximately $10 \%$ of objects cannot be interpreted (category "D"). And about $50 \%$ of objects can be interpreted using additional materials. In the presence of even additional materials, difficulties arise when solving the problem of interpreting 
various types of objects that have small sizes of point and short-length objects.

The result of interpreting images obtained from on-board optical-electronic surveillance systems depends on the quality of image segmentation. Especially taking into account the features of their preparation, such as a heterogeneous background, variability of various parts of the image, the presence of noise) [9]-[15]. It is not always possible to ensure the stability of segmentation methods to variations of different image parameters when using known image segmentation methods.

\subsection{Problem analysis}

It is known [15]-[17] that the main techniques for image segmentation are divided into:

1. Subjective.

2. Objective.

2.1. Systemic

2.2. Direct.

2.2.1. Analytical.

2.2.2. Empirical.

2.2.2.1. Controllable.

2.2.2.2. Uncontrolled (automatic).

One of the key elements of the technique of comparative testing is the criterion for assessing the quality of image segmentation. Currently, the main criteria and indicators have been developed for two main approaches to image segmentation [16]-[19]:

1) dividing the image by contours into areas with similar characteristics (edge-based methods, boundary-based, contour-based);

2) combining image pixels into groups based on the proximity of some quantitative features (region-based methods).

For estimation the results of the methods of the first group, the same ones are used as for boundary detectors (such indicators are given in [11]). The criteria estimation of the quality of image segmentation methods related to the second group are given in [16]. The main features of high-quality segmentation are [16]:

- uniformity of the area in terms of characteristics (first of all, in terms of color and texture);

- the difference in the values of the selected characteristics for adjacent areas of the image;

- smoothness of borders of each image segment;

- a small number of "holes" in the segment.

According to the correspondence to the indicated features, the known indicators of image segmentation quality are classified [16].

1. The first group - the indicators that are based on a comparison with the reference segmentation.

1.1. The number of pixels assigned during segmentation is not up to its segment. It is estimated by constructing an imprecision matrix (table 2). The columns of the matrix correspond to the class the pixels really belong to. Matrix rows correspond to the class to which the pixels are assigned during segmentation. Thus, correctly classified pixels refer to matrix elements located on the main diagonal. Wrongly classified pixels refer to all other elements of the matrix.

The indicators used in this are as follows. The first is the percentage of incorrectly classified pixels of this class to the total number of pixels of this class on the reference image (expression (1)):

$$
M_{1}^{k}=100 \frac{\left(\sum_{i=1}^{n} C_{i k}\right)-C_{k k}}{\sum_{i=1}^{n} C_{i k}},
$$

where $n-$ the number of classes; $C_{k k}-$ the number of correctly classified pixels of the $k$-th class; $\sum_{i=1}^{k} C_{i k}-$ the number of pixels really belong to $k$-th class.

Table 2: The example of matrix of inaccuracy []

\begin{tabular}{|c|c|c|c|c|c|c|}
\hline & BK & PA & RD & CY & NU & Total \\
\hline BK & 909 & 2 & & 2 & & 913 \\
\hline PA & & & & & & \\
\hline RD & & & 111 & 10 & & 121 \\
\hline CY & 37 & 3 & 67 & 802 & 1 & 910 \\
\hline NU & & & & 87 & 419 & 506 \\
\hline Total & 946 & 5 & 178 & 901 & 420 & \\
\hline
\end{tabular}

The second indicator is the percentage of pixels mistakenly assigned to the $k$-th class to the total number of pixels of the second classes on the reference image (expression (2)):

$$
M_{2}^{k}=100 \frac{\left(\sum_{i=1}^{n} C_{k i}\right)-C_{k k}}{\left(\sum_{i=1}^{n} \sum_{k=1}^{n} C_{i k}\right)-\sum_{i=1}^{n} C_{i k}},
$$

where $\sum_{i=1}^{n} C_{k i}-$ the number of pixels that are assigned to the $k$-th class; $\sum_{i=1}^{n} \sum_{k=1}^{n} C_{i k}-$ the total number of pixels in the picture of the $k$-th class; $C_{k k}-$ the number of correctly classified pixels of the $k$-th class; $\sum_{i=1}^{k} C_{i k}$ - the number of pixels really belong to $k$-th class. 
The third indicator is the probability of error of segmentation $p($ err $)$. The probability that the pixel of the original image will be attributed to the object $p_{s}(o)$ on the segmented image can be represented by the expression (3):

$$
p_{s}(o)=\mathrm{p}(o) \mathrm{p}(o / o)+\mathrm{p}(b) \mathrm{p}(o / b),
$$

where $p(o), p(b)$ - the probabilities that a randomly selected pixel of the original image belongs to the object or background, while $p(o)+p(b)=1 ; p(o / o)$ - the probability that the pixel that belongs to the object during segmentation will also be attributed to the object; $p(o / b)$ the probability that a pixel that belongs to the background during segmentation will be mistakenly assigned to an object.

The probability that the pixel of the original image will be assigned to the background on the segmented image can be represented by the expression (4):

$$
p_{S}(b)=\mathrm{p}(b) \mathrm{p}(b / b)+\mathrm{p}(o) \mathrm{p}(b / o)
$$

where $p(b / b)$ - the probability that a pixel belonging to the background will also be referred to the background during segmentation; $p(b / o)$ - the probability that a pixel belonging to the object during segmentation will be erroneously assigned to the background.

The probability is determined by the expression (5):

$$
p(e r r)=\mathrm{p}(o) \mathrm{p}(b / o)+\mathrm{p}(b) \mathrm{p}(o / b)
$$

1.2. Indicators that characterize the incorrect location of classified pixels. The first indicator is (expression (6)):

$$
\varepsilon=\frac{\sqrt{\sum_{i=1}^{N} d_{i}^{2}}}{A} \times 100
$$

where $N$ - the number of erroneously classified pixels; $A$ the total number of pixels on the image; $d_{i}$ - the Euclidean distance between the $i$-th erroneously classified pixels, which really refers to this class.

The range of values takes $\varepsilon$ from 0 (with perfect segmentation) to $\varepsilon_{\max }$, which depends on the image size $n$. And calculated for area image of the size $(n \times n)$ according to the expression (7):

$$
\varepsilon_{\max }=\left\{\begin{array}{c}
100 \sqrt{\frac{7}{6}-\frac{3}{2 n}+\frac{1}{3 n^{2}},} \text { if } n-\text { even } \\
100 \sqrt{\frac{7}{6}-\frac{3}{2 n}-\frac{1}{6 n^{2}}+\frac{1}{2 n^{2}}}, \text { if } n \text {-odd }
\end{array}\right.
$$

The second indicator - FOM (figure of merit) - is the empirical distance of a given pixel from its actual location.

There are two types of FOM indicator - expressions (8) and (9):

$$
\begin{gathered}
\text { FOM }=\frac{1}{N} \sum_{i=1}^{N} \frac{1}{1+\gamma d_{i}^{2}}, \\
\text { FOM }_{1}=\left\{\begin{array}{c}
\frac{1}{N_{e}} \sum_{i=1}^{N} \frac{1}{1+\gamma d_{i}^{2}}, N_{e}>0, \\
1, N_{e}=0 .
\end{array}\right.
\end{gathered}
$$

where $N$ - the number of pixels on the image; $d_{i}-$ the distance of the $i$-th pixel of the image to the nearest pixel assigned to the same class in the image reference; $\gamma-$ the scale factor; $N_{e}$ - pixels are erroneously classified.

There are several more empirical indicators of the quality of image segmentation, for example, - the Hausdorff dimension (expression (10)):

$$
\text { Hausdorff }\left(I_{t}, I_{s}\right)=\max \left(h\left(I_{t}, I_{s}\right), h\left(I_{s}, I_{t}\right)\right),
$$

where $I_{t}, I_{s}$ - the sets of points of different areas; $h\left(I_{s}, I_{t}\right)$ - the distance between the respective areas;

$$
h\left(I_{t}, I_{S}\right)=\max _{t_{i} \in I_{t}} \min _{s_{i} \in I s}\left\|t_{i}=s_{i}\right\| .
$$

If so $h\left(I_{t}, I_{s}\right)=d$, then this means that all the pixels of the set $I_{t}$ are no further than at a distance $d$ from the set of the pixels $I_{s}$.

They also use indicators [16]:

1) RMS (root mean squared error) - observational error (expression (12)):

$$
R M S\left(I_{1}, I_{2}\right)=\left[\frac{1}{\operatorname{card}(X)} \sum_{x \in X}\left(I_{1}(x)-I_{2}(x)\right)^{2}\right]^{\frac{1}{2}}(12)
$$

where $\operatorname{card}(X)-$ the number of pixels in a set $X ; I_{i}(X)-$ intensity the pixel $x$ in $I_{i} ; X$ - the set of pixels on the segmented image; 
2) the Baddeley exponent (expression (13)):

$\operatorname{Baddeley}\left(I_{1}, I_{2}\right)=\left[\frac{1}{\operatorname{card}(X)} \sum_{x \in X}\left|d\left(x, I_{1}\right)-d\left(x, I_{2}\right)\right|^{p}\right]^{\frac{1}{p}}$,

where $d(x, I)=\min _{y \in I} d(x, y) ; p \geq 1$,

and other. Indicators according to expressions (8)-(13) are also called supervisory indicators for assessing the quality of image segmentation [16].

1.3. Indicators that characterize the degree of image fragmentation (expression (14)):

$$
F R A G=\frac{1}{1+\left|\alpha\left(n_{R}-n_{1}\right)\right|^{\beta}},
$$

where $n_{R}$ - the number of segments in the segmented image; $n_{R}$ - the number of segments in the reference image; $\alpha, \beta$ - scale factors.

1.4. Indicators that characterize the values of the characteristics of the original image used for segmentation (expression (15)):

$$
F O C=\frac{1}{N} \sum_{i=1}^{N} \frac{1}{1+\left|\psi\left(f_{i}-\mu_{j}\right)\right|^{\delta}},
$$

where $N$ - the number of pixels in the image; $f_{1}$ - the value of the intensity of the pixel and the original image; $\mu_{j}-$ the representative value of the intensity of the $j$-th segment, to which the $i$ pixel was assigned during segmentation; $\psi, \delta$ - scale parameters.

2. The second group - indicators that do not require a reference segmentation.

2.1. The indicator that take into account the homogeneity of the segments. It is based on calculating the variance of the value of the corresponding feature of the image, is used for segmentation.

Let be $f_{i}$ - the value of the feature $F$ in pixels $i$. Then the variance of the feature $F$ for the segment of the image $R_{j}$ is determined as follows:

$$
\sigma_{j}^{2}=\sum_{i \in R_{j}} \frac{\left(f_{i}-\bar{f}_{j}\right)^{2}}{A_{j}},
$$

where $\bar{f}_{j}=\sum_{i \in R_{j}} \frac{f_{i}}{A_{j}} ; A_{1}-$ the area of the segment.

The degree of homogeneity of the area $\Omega$, which consists of $R_{j}$ segments, is determined by the expression (17):

$$
U_{\Omega}=1-\sum_{R_{i} \in \Omega} \frac{w_{j} \sigma_{j}^{2}}{N},
$$

where $w_{j}$ - the weight that determines the segment's contribution $R_{j}$ to $U_{\Omega} ; N=\delta_{\max }^{2} \sum_{R_{j} \in \Omega} w_{j}-$ normalizing coefficient; $\delta_{\max }^{2}=\frac{1}{2}\left(f_{\max }-f_{\min }\right)^{2} ; f_{\max }, f_{\min }-$ the maximum and minimum value of the feature $F$ in the area $\Omega$.

2.2. An indicator that takes into account the contrast between segments.

$$
c_{i j}=\frac{\left|\bar{f}_{i}-\bar{f}_{j}\right|}{\bar{f}_{i}+\bar{f}_{j}},
$$

where $\bar{f}_{\mathrm{i}}, \bar{f}_{j}$ - the average values of the feature $F$ in segments $R_{i}$ and $R_{j}$, respectively.

2.3. A complex indicator, which, for example, takes into account both the homogeneity of the segments and their number (expression (19)):

$$
F=\frac{1}{1000 N} \sqrt{R} \sum_{i=1}^{R} e_{i}^{2} / \sqrt{A_{i}},
$$

where $N$ - the number of pixels on the image; $R-$ the number of segments; $A_{i}$ - the area of the $i$-th segment; $e_{i}$ a value that characterize the degree of homogeneity of the $i$-th segment.

However, the above indicators for assessing the quality of image segmentation have the following disadvantages:

- sometimes the segmentation results, which are the best from the experts' point of view, have a higher percentage of erroneously classified mudflow peaks;

- the location of false points relative to the corresponding segment is not taken into account. Therefore, the error at the boundary and the error at the center of the segment should be penalized differently;

- the difference in the importance of individual image areas for segmentation is not taken into account. Errors for different image segments should have different weights;

- there is no information about the class of pixels. This introduces the biggest error.

Thus we will choose the Canny edge detection algorithm as a segmentation method. This method is optimal in the following parameters:

- the criterion for selecting contours - the method should select as many borders as possible on the image;

- the localization of edges - outlines, highlighted, should be located as close to the border on the image as possible;

- the minimizing the number of reviews of one edge - each circuit should be marked once and, if possible, the contours should not be created due to noise. 
We will choose the Kullback-Leibler divergence as an indicator of efficiency of segmentation.

\section{MAin Material}

In the papers of Canny, for example [6], the concept of Non-Maximum Suppression was introduced. It means that the border pixels are the points at which the gradient reaches its maximum in the direction of the gradient vector. The steps of the Canny method are outlined below.

1. Smoothing. It is carried out in order to reduce the influence of noise on the definition of boundaries. For this, a Gaussian filter was used (expression (20)):

$$
f(x, y)=\frac{1}{2 \pi \sigma} \mathrm{e}^{\left(-\frac{x^{2}+y^{2}}{2 \sigma^{2}}\right)},
$$

where $(x, y)$ - the coordinates of the pixel on the image; $f(x, y)$ - the brightness of image; $\sigma$ - the blur parameter.

The value of the blur parameter must be selected to provide more noise reduction. A larger value of the parameter is used to highlight large boundaries, less to highlight small details.

2. Finding a gradient. To determine the gradient in the image after the Gaussian filter (20), we will use the Sobel operator [6]. the spatial filtering scheme using the Sobel operator is shown in Figure 1.

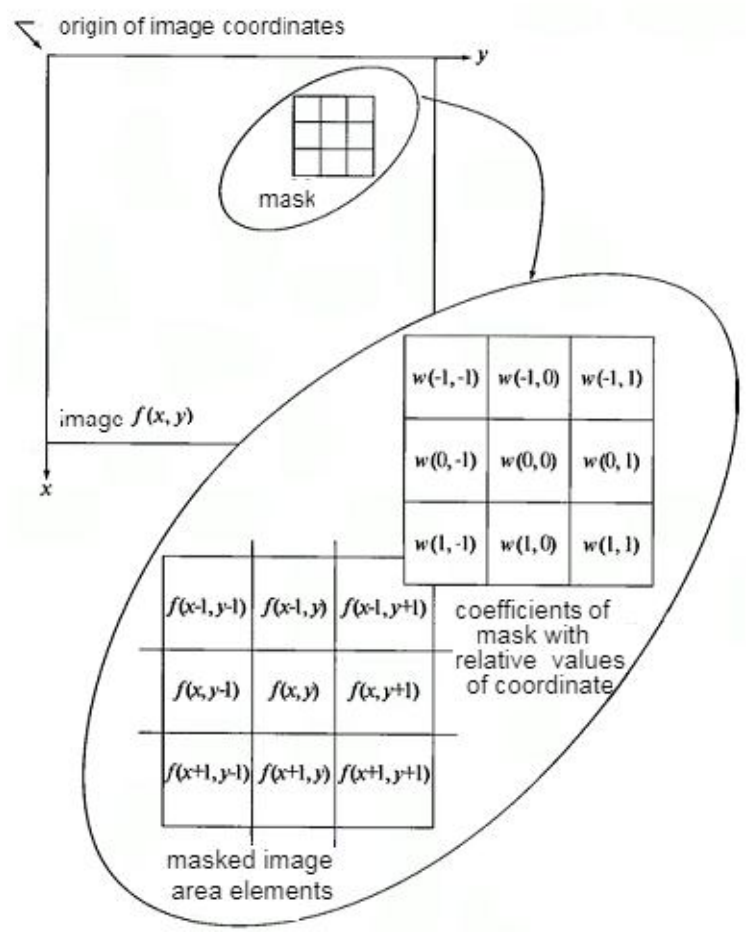

Figure 1: The scheme of spatial filtering with using the Sobel operator
The Sobel transformation is based on the assumption that the brightness discontinuity function at the edges becomes much larger. From this assumption, we can draw the following conclusion: to find the faces, it is enough to differentiate the brightness function $f(x, y)$ (expressions (21), (22)):

$$
\begin{aligned}
& \frac{\partial f(x, y)}{\partial x}=\Delta x=\frac{f(x+d x, y)-f(x, y)}{d x}, \\
& \frac{\partial f(x, y)}{\partial y}=\Delta y=\frac{f(x, y+d y)-f(x, y)}{d y},
\end{aligned}
$$

In discrete images $d x$ and $d y$ can be measured in the number of pixels between two points using expressions (23), (24):

$$
\begin{aligned}
& \Delta x=f(i+1, j)-f(i, j), \\
& \Delta y=f(i, j+1)-f(i, j),
\end{aligned}
$$

The expression for determining the magnitude of the gradient $G$ can be written as follows (expression (25)):

$$
G=\sqrt{(\Delta x)^{2}+(\Delta y)^{2}},
$$

and the direction $\theta$ (expression (26):

$$
\theta=\arctan \left(\frac{\Delta y}{\Delta x}\right)
$$

In expressions (25), (26), the gradient is estimated using masks $(2 \times 2)$ :

$$
\begin{aligned}
& \Delta x=\left(\begin{array}{cc}
-1 & 1 \\
0 & 0
\end{array}\right), \\
& \Delta y=\left(\begin{array}{cc}
-1 & 0 \\
1 & 0
\end{array}\right) .
\end{aligned}
$$

The main disadvantage of using masks (27) and (28) is a large number of errors due to the presence of noise. In addition, the use of even-order masks makes it impossible to evaluate based on the pixel that is located in the center of the mask. Therefore, we will use the Sobel operator with masks (29) and (30):

$$
\begin{aligned}
K_{G_{x}} & =\left(\begin{array}{ccc}
-1 & 0 & 1 \\
-2 & 0 & 2 \\
-1 & 0 & 1
\end{array}\right), \\
K_{G_{y}} & =\left(\begin{array}{ccc}
1 & 2 & 1 \\
0 & 0 & 0 \\
-1 & -2 & -1
\end{array}\right) .
\end{aligned}
$$

From the analysis of expressions (29), (30) (in comparison with (27), (28)), one can see the use of the coefficient 2 for the 
average elements. The increased factor value is used to reduce the anti-aliasing effect by giving more weight to the midpoints. The value and direction of the gradient take the form (31), (32), respectively:

$$
\begin{gathered}
G=\sqrt{G_{x}^{2}+G_{y}^{2}}, \\
\theta=\arctan \left(\frac{\left|G_{x}\right|}{\left|G_{y}\right|}\right) .
\end{gathered}
$$

After using the Sobel operator, the intensity of each pixel in the original image is equal to the gradient of the brightness vector.

3. Suppression of false maxima. The purpose of this stage is to turn the "blur" boundaries into "clear". This is achieved by keeping local maxima and removing everything else. For each pixel, the following actions are performed:

- the direction of the gradient is rounded to the nearest multiple of $45^{\circ}$ (Figure 2a);

- if a local maximum in the direction of the gradient is reached at the current point, then it is part of the boundary;

- otherwise, the point is deleted (Figure 2b).

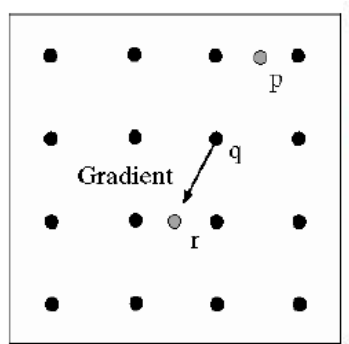

a)

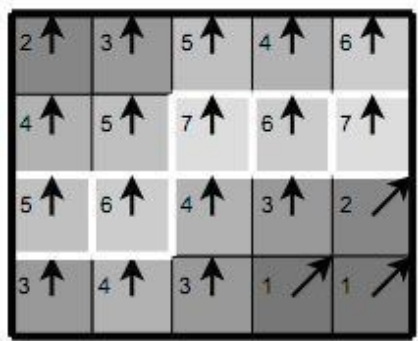

b)
Figure 2: Search for local maxima: a) $p$ and $r$ maxima are interpolated (deleted); b) principle of suppression of false maxima

The principle of suppression is illustrated in Figure 2b. All pixels in Figure $2 b$ have an "upward orientation", therefore, the gradient value at these points will be compared with lower and higher located pixels. Pixels circled in white in Figure $2 b$ will remain in the original image. Others will be suppressed.

4. Double threshold filtration (Figure 3). The goal is every pixel that exceeds the upper threshold is marked as "strong". Each pixel that falls between the two thresholds is "weak" (the brightness of such points takes a fixed average value and will be refined at the next stage). Pixels less than the lower threshold are removed. Using a double threshold allows you to reduce the effect of noise (due to the upper threshold) and not lose "tails" (due to the lower threshold).

5. Tracing the area of uncertainty. The task is reduced to the selection of groups of pixels that received an intermediate value at the previous stage. And assigning them to the border (if they are connected to one of the established borders) or suppressing them (otherwise).

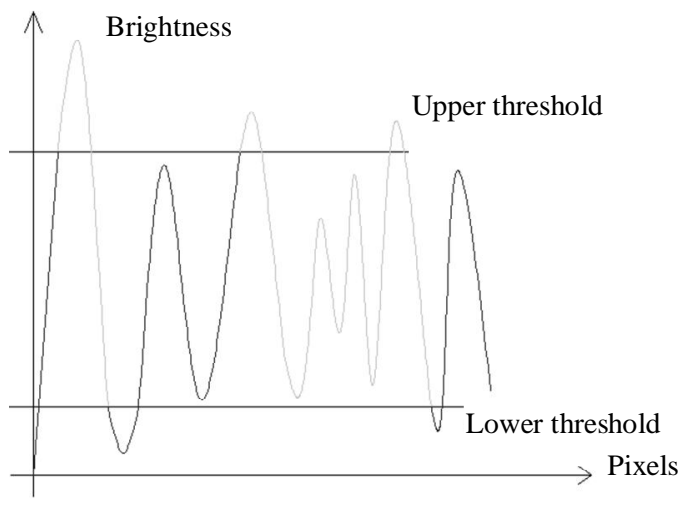

Figure 3: Using two thresholds

The result of segmentation of the original image (Figure 4) by the Canny method is shown in Figure 5.

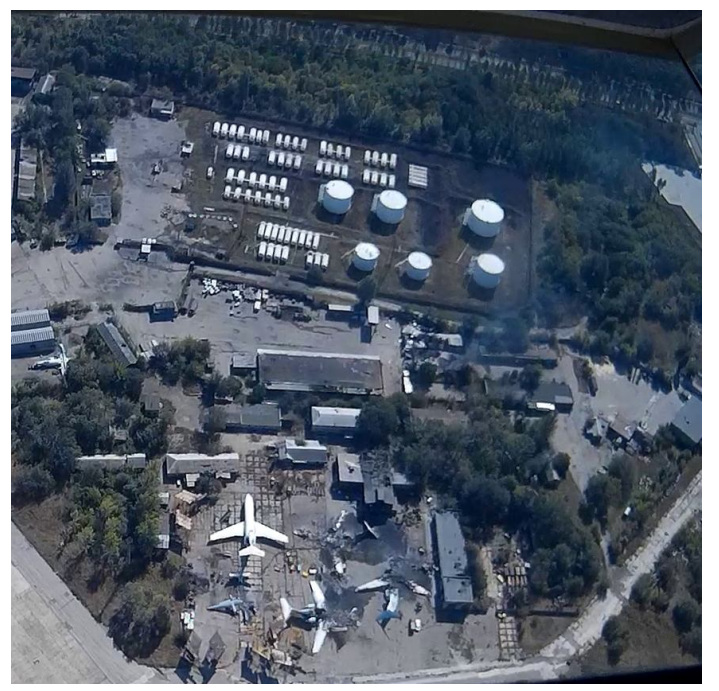

Figure 4: The original image [20]

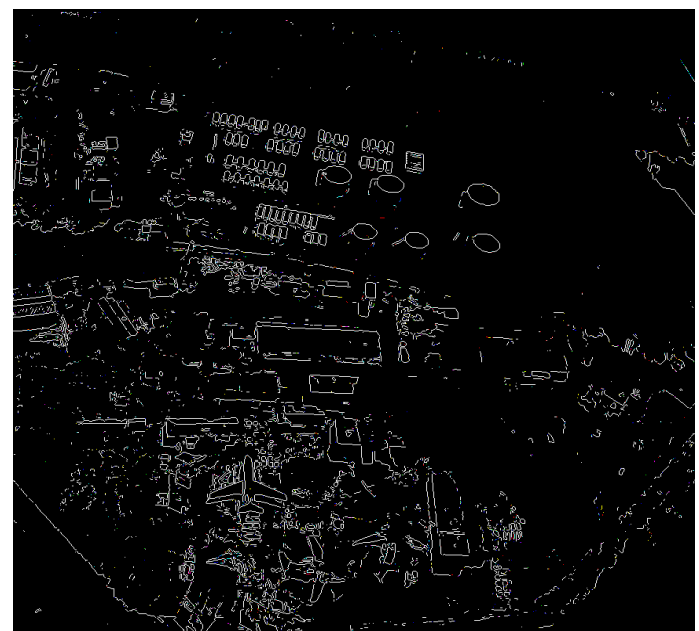

Figure 5: The result of segmentation of the original image (Figure 4) by the Canny method 
As an indicator of the quality of segmentation of the original image (Fig. 4) by the Canny method, we select the information indicator - the Kullback-Leibler divergence (expression (33)):

$$
K\left(p_{\xi}, p_{\eta}\right)=\int_{R^{2}} p_{\xi}(x) \log \frac{p_{\xi}(x)}{p_{\eta}(x)} d x,
$$

where $p_{\xi}$ - the distribution of brightness on the original image (Figure 4); $p_{\eta}$ - the distribution of brightness on the segmented image (Figure 5); $R^{2}$ - the area of the image.

Figure 6 shows the curve of the dependence of the Kullback-Leibler divergence on the change in the scaling factor of the original image (Figure 4) during image segmentation using the Canny method.

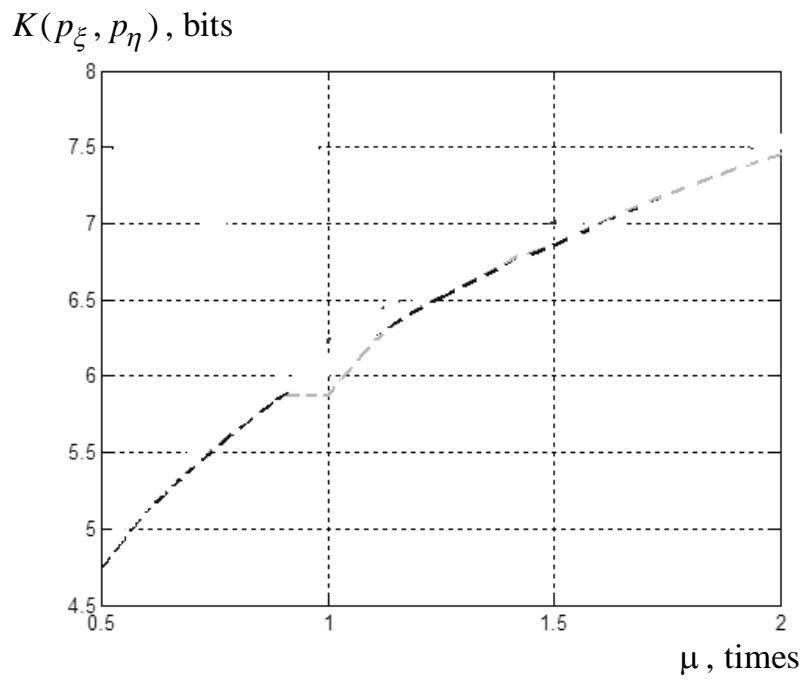

Figure 6: The dependence of the Kullback-Leibler divergence on changes in the scaling factor of the original image (Figure 4) during image segmentation using the Canny method

The Kullback-Leibler divergence was calculated using expression (33) using the base 2 logarithm. Therefore, the Kullback-Leibler divergence is measured in bits. And for the image in gradations $(0 \ldots 255)$, the maximum value of the Kullback-Leibler divergence is 8 .

\section{CONCLUSION}

Thus, it was found that the result of interpretation of images obtained from on-board optoelectronic surveillance systems depends on the quality of image segmentation. This especially depends on the features of their preparation, namely, on a heterogeneous background, variability of various parts of the image, and the presence of noise. The main techniques, criteria and indicators of image segmentation, their advantages and disadvantages have been analyzed.
It is proposed to evaluate the information indicator (the Kullback-Leibler divergence) of optoelectronic image segmentation using the Canny method.

The analysis of the main stages of the Canny method is carried out: smoothing, finding a gradient, suppression of false maxima, double threshold filtration, tracing the area of uncertainty. The results of the segmentation of the optoelectronic image by the Canny method are presented. The Kullback-Leibler divergence is calculated for its dependence on the scale factor of the original image.

The direction of further research is to compare different segmentation methods using an informational indicator - the Kullback-Leibler divergence.

\section{REFERENCES}

1. I. Ruban, H. Khudov, V. Lishchenko, A. Zvonko, S. Glukhov, I. Khizhnyak, V. Maliuha, Y. Polonskyi, R. Kushpeta, The Calculating Effectiveness Increasing of Detecting Air Objects by Combining Surveillance Radars into The Coherent System, International Journal of Emerging Trends in Engineering Research, Vol. 8., № 4, 2020, pp. 1295-1301. https://doi.org/10.30534/ijeter/2020/58842020.

2. H. Khudov, I. Khizhnyak, I. Yuzova, O. Baranik, G. Semiv, S. Bondarenko, and O. Tytarenko. The Optimization Technique for Joint Discrete Search and Detection of Observation Objects, International Journal of Emerging Trends in Engineering Research, № 8(2), 2020, pp. 533-538. https://doi.org/10.30534/ijeter/2020/42822020.

3. V. Lishchenko, V. Chaliy, H. Khudov, and A. Zvonko. Proposals for Improving of Air Surveillance Informativity in MIMO Radar Systems Based on Two-Dimensional Radars, in Intern. Scient.-Pract. Conf. Problems of Infocommunications. Science and Technology (PIC S\&T), 2018, pp. 153-156. https://doi.org/10.1109/infocommst.2018.8632052.

4. H. Khudov, A. Zvonko, I. Khizhnyak, V. Shulezko, V. Khlopiachyi, V. Chepurnyi, and I. Yuzova. The Synthesis of the Optimal Decision Rule for Detecting an Object in a Joint Search and Detection of Objects by the Criterion of Maximum Likelihood, International Journal of Emerging Trends in Engineering Research, № 8(2), 2020, pp. 520-524. https://doi.org/10.30534/ijeter/2020/40822020.

5. H. Khudov, S. Glukhov, V. Podlipaiev, V. Pavlii, I. Khizhnyak, and I. Yuzova The Multiscale Image Processing Method from On-board Earth Remote Sensing Systems Based on the Artificial Bee Colony Algorithm, International Journal of Advanced Trends in Computer Science and Engineering, Vol. 9. № 3, 2020, pp. 2557-2562.

https://doi.org/10.30534/ijatcse/2020/11932020.

6. I. Ruban, and H. Khudov, Advances in Spatio-Temporal Segmentation of Visual Data, Chapter 2. Swarm 
Hennadii Khudov et al., International Journal of Emerging Trends in Engineering Research, 8(7), July 2020, 3927 - 3934

Method of Image Segmentation. Series Studies in Computational Intelligence (SCI), Vol. 876. - Publisher Springer, Cham, $2020 . \quad-\quad$ P. 53-99. DOI https://doi.org/10.1007/978-3-030-35480-0.

7. J.L. Bayuk, J. Healey, P. Ronmeyer, M.H. Sachs, J. Schmidt, and J. Weiss, Cyber security policy guidebook. - New Jersey.: Wiley. - 2017. - 270 p.

8. S. Pisupati and M. Ismail, Image Registration Method for Satellite Image Sensing using Feature based Techniques, International Journal of Advanced Trends in Computer Science and Engineering, Vol. 9. № 1, 2020, pp. 590-593.

https://doi.org/10.30534/ijatcse/2020/82912020.

9. I. Ruban, V. Khudov, O. Makoveichuk, H. Khudov, and I. Khizhnyak. A Swarm Method for Segmentation of Images Obtained from On-Board Optoelectronic Surveillance Systems, in Intern. Scient.-Pract. Conf. Problems of Infocommunications. Science and Technology (PIC S\&T), 2018, pp. 613-618. DOI: https://doi.org/10.1109/infocommst.2018.8632045.

10. I. Ruban, H. Khudov, O. Makoveichuk, M. Chomik, V. Khudov, I. Khizhnyak, V. Podlipaiev, Y. Sheviakov, O. Baranik, and A. Irkha. Construction of methods for determining the contours of objects on tonal aerospace images based on the ant algorithms, Eastern-European Journal of Enterprise Technologies, № 5/9 (101), 2019, pp. 25-34.

https://doi.org/10.15587/1729-4061.2019.177817.

11. I. Ruban, H. Khudov, O. Makoveichuk, I. Khizhnyak, V. Khudov, V. Podlipaiev, V. Shumeiko, O. Atrasevych, A. Nikitin, and R. Khudov. Segmentation of opticalelectronic images from on-board systems of remote sensing of the Earth by the artificial bee colony method, Eastern-European Journal of Enterprise Technologies, № 2/9 (98), 2019, pp. 37-45. https://doi.org/10.15587/1729-4061.2019.161860.

12. I. Ruban, V. Khudov, H. Khudov, and I. Khizhnyak An Improved Method for Segmentation of a Multiscale Sequence of Optoelectronic Images, in Intern. Scient.-Pract. Conf. Problems of Infocommunications. Science and Technology (PIC S\&T), 2017, pp. 137-140. https://doi.org/10.1109/INFOCOMMST.2017.8246367.

13. I. Ruban, O. Makoveichuk, V. Khudov, I. Khizhnyak, H. Khudov, I. Yuzova, and Y. Drob. The Method for Selecting the Urban Infrastructure Objects Contours, in Intern. Scient.-Pract. Conf. Problems of Infocommunications. Science and Technology (PIC S\&T), 2019, pp. 689-693. DOI: https://doi.org/10.1109/infocommst.2018.8632045.

14. H. Khudov, O. Makoveychuk, I. Khizhnyak, I. Yuzova , A. Irkha, and V. Khudov. The Mosaic Sustainable Marker Model for Augmented Reality Systems, IJATCSE, № 9(1), 2020, pp. 637-642. https://doi.org/10.30534/ijatcse/2020/89912020.

15. H. Khudov, I. Ruban, O. Makoveichuk, H. Pevtsov, V. Khudov, I. Khizhnyak, S. Fryz, V. Podlipaiev, Y. Polonskyi, and R. Khudov. Development of methods for determining the contours of objects for a complex structured color image based on the ant colony optimization algorithm, Eureka: Physics and Engineering, № 1, 2020, pp. 34-47. DOI: https://doi.org/10.21303/2461-4262.2020.001108.

16. I. Ruban, H. Khudov, V. Khudov, I. Khizhnyak, and O. Makoveichuk. Segmentation of the images obtained from onboard optoelectronic surveillance systems by the evolutionary method, Eastern-European Journal of Enterprise Technologies, № 5/9 (89), 2017, pp. 49-57. DOI: https://doi.org/10.15587/1729-4061.2017.109904.

17. I. Ruban, H. Khudov, O. Makoveichuk, I. Khizhnyak, N. Lukova-Chuiko, G. Pevtsov, Y. Sheviakov, I. Yuzova, Y. Drob, and O. Tytarenko, Method for determining elements of urban infrastructure objects based on the results from air monitoring, Eastern-European Journal of Enterprise Technologies, № 4/9 (100), 2019, pp. 52-61.

https://doi.org/10.15587/1729-4061.2019.174576.

18. V. Lishchenko, H. Khudov, V. Tiutiunnyk, V._Kuprii, F._Zots, and G._Misiyuk The Method of Increasing the Detection Range of Unmanned Aerial Vehicles In Multiradar Systems Based on Surveillance Radars, in 2019 IEEE 39th International Conference on Electronics and Nanotechnology (ELNANO), 2019. P. 559-562. https://doi.org/10.1109/ELNANO.2019.8783263.

19. V. Lishchenko, H. Khudov, B. Lisogorsky, O. Baranik, D. Holovniak, and O. Serdjuk The MIMO System on Based Existing Mechanical Rotation Radars with Wide Surveillance Area, in 2020 IEEE 40th International Conference on Electronics and Nanotechnology (ELNANO), 2020. P. 625-628. DOI: https://doi.org/10.1109/ELNANO.50318.2020.90887463

20. Ikonos Satellite Image Gallery, URL: http://www.satimagingcorp.com/gallery/ikonos/ (accessed 12 November 2019). 